\title{
ARTÍCULO
}

\section{A vueltas con el Transhumanismo: cuestiones de futuro imperfecto Dealing with Transhumanism: Issues about the Future Imperfect}

\author{
Roger Campione \\ Departamento de Ciencias Jurídicas Básicas \\ Universidad de Oviedo
}

Fecha de recepción 31/01/2019 | De aceptación: 03/06/2019 | De publicación: 27/06/2019

\section{RESUMEN,}

El Transhumanismo es una perspectiva que aboga por la posibilidad de mejorar la condición humana física, emocional y cognitiva utilizando el progreso y la tecnología. Las cuestiones que plantea requieren asumir dos premisas, una normativa y otra empírica: la primera es que la formación para el uso de las tecnologías requiere una educación ética y jurídica, además de científica. La segunda implica que en un futuro próximo habrá robots muy sofisticados y dotados de inteligencia artificial avanzada que podrán tomar decisiones operativas, así que ¿cómo regular estas capacidades? Las aplicaciones en el ámbito sanitario muestran los términos del trilema normativo relativo a la distinción conceptual entre "terapia", "mejora” y "superación” de la condición humana.

\section{PALABRAS CLAVE.}

Transhumanismo, tecnologías convergentes, perfeccionamiento humano, inteligencia artificial y derecho.

\section{ABSTRACT.}

Transhumanism stands up for the physical, emotional and cognitive human enhancement by progress and technology. The issues in question involve both a normative and an empirical assumption: the first one undertakes that the use of new technologies demands not only a scientific approach but also an ethical and legal one. The second means that in the near future advanced robots with artificial intelligence will make working decisions. So, how shall we have to rule those abilities? Many health implementations show the normative trilemma posed by the conceptual difference between therapy, enhancement and going beyond the human condition.

\section{KEY WORDS.}

Transhumanism, Converging Technologies, Human Enhancement, AI and Law. 
Sumario: 1. Introducción; 2. Viejos dilemas y nuevos retos; 3. Las implicaciones normativas de las tecnologías convergentes; 4. Transhumanismo y salud

Trasumanar significar per verba non si poria; però l'essemplo basti a cui esperienza grazia serba

\section{Introducción}

Antes de hablar del Transhumanismo como sistema o conjunto de ideas, conviene una aclaración filológica: el prefijo "trans" significa, en su etimología latina, "más allá de" o "a través de". Es una partícula que indica el pasar más allá de un umbral, un atravesamiento, la mutación de una condición a otra, etc. La definición del sustantivo "humanismo", en cambio, es un poco más compleja y articulada porque engloba diferentes ramificaciones: es un renacimiento del ser humano entendido como proceso de renovación cultural que busca en las fuentes clásicas una nueva forma de pensamiento. Entraña la exigencia de reconocer la dimensión histórica de los acontecimientos, ignorada por la Edad Media. El humanismo es, en este sentido, una perspectiva. Para ello resulta esencial la investigación filológica, para recuperar en ella el significado auténtico de los clásicos. Pero el humanismo también es "renacentista" porque re-descubre el valor del hombre como ser mundano, insertado en el mundo natural y capaz de forjar su propio destino. Esta condición renovada se afirma en la capacidad del ser humano de proyectar su existencia dentro de las coordenadas históricas y naturales. Finalmente, un componente fundamental del humanismo es la exigencia de la tolerancia religiosa basada en la unidad filosófica de todas las religiones aun en la diversidad de sus cultos ${ }^{1}$. Es desde aquí que debería empezar a hablarse de transhumanismo como fórmula actual de una doctrina que apuesta por la posibilidad de mejorar la condición humana gracias a la hibridación entre nuestra existencia natural y la inteligencia artificial.

Y es curioso que el vocablo, en forma de verbo, lo utilice por primera vez precisamente uno de los primigenios y más destacados humanistas, Dante Alighieri, en el Canto I del Paraíso, en los versículos que hacen de exergo a este $\operatorname{artículo}^{2}$ : no se podría expresar con palabras la superación de la

\footnotetext{
${ }^{1}$ Abbagnano, N., Storia della filosofía 2. La filosofia moderna: dal Rinascimento all 'Illuminismo, Turín, UTET, 1993, pp. 4-9

${ }^{2}$ Nick Bostrom señala que el primero que parece haber utilizado la palabra "transhumanismo" en su significado actual fue en 1927 el biólogo Julian Huxley, hermano de Aldous, en Religion without revelation (Bostrom, N., "A History of Transhumanist Thought", Journal
} 
condición humana, así que el ejemplo que acabo de poner sea suficiente para los que reciban por gracia divina la experiencia de semejante condición. Trasumanar es un neologismo que Dante inventa para expresar lo que siente al ascender con Beatriz del paraíso terrenal a las esferas celestes ${ }^{3}$. Lo que quiere decir es que elevarse más allá de los límites de la naturaleza, es decir, pasar de lo humano a un grado más alto, que no puede ser sino divino, es algo que no se puede explicar con palabras. Evidentemente, ni el humanismo ni el transhumanismo actual expresan esa condición en relación con Dios, tal como entendía el Sommo Poeta, pero el ejemplo de Dante nos vale perfectamente para introducir los asuntos del transhumanismo ${ }^{4}$. Porque en esos versos de la Divina Comedia Dante se está refiriendo al ejemplo de Glauco, el mítico pescador de Beocia que habiendo probado una hierba milagrosa se convirtió en una divinidad inmortal ${ }^{5}$. Y uno de los máximos representantes del transhumanismo actual, Nick Bostrom, recurre a un arquetipo muy parecido, el rey sumerio Gilgamesh -que habiendo partido a la búsqueda de la inmortalidad descubrió su secreto en una hierba que crecía en el fondo del mar- para situar ya en la remota antigüedad el deseo humano de adquirir nuevas capacidades expandiendo los términos de nuestra existencia y nuestras posibilidades de actuar ${ }^{6}$. Muchos fueron los símbolos de esta “mitología de la superación”, como Dédalo, que usó su inteligencia para trascender los límites impuestos al hombre por la naturaleza, o como Prometeo, que retando a los dioses robó el fuego para mejorar al ser humano.

Y con la mejora también empieza la inquietud, pues no es dado saber con certeza adónde nos llevarán la razón y la técnica. Visto desde el Olimpo, ese fue el error de Prometeo, no haber tenido en cuenta que sin la sabiduría política, sin una idea acerca de lo correcto, el dominio de la técnica surte

of Evolution and Technology, Vol. 14, 2005, pp. 1-25, publicado sucesivamente en Argumentos de razón técnica, n. 14, 2011, versión por la que se cita, pp. 157-191). En la literatura española hay quien señala como obra precursora Dédalo; Ciencia y futuro, de J.B.S. Haldane (de Asís, R., Una mirada a la robótica desde los derechos humanos, Madrid, Dykinson, 2015, p. 52) y quien se remonta hasta La nueva Atlántida de Francis Bacon y, en términos muy generales, incluso al Renacimiento (Diéguez, A., Transhumanismo. La búsqueda tecnológica del mejoramiento humano, Barcelona, Herder, 2017, pp. 30-31).

${ }^{3}$ Cfr. Eliot, T.S., La aventura sin fin. Ensayos, ed. de A. Jaume, Madrid, Lumen, 2011.

${ }^{4}$ Aun así, no faltan, como se ha señalado, los que entienden el transhumanismo como una nueva religión y la propia religión como una forma prematura de transhumanismo (vid. Diéguez, A., Transhumanismo. La búsqueda tecnológica del mejoramiento humano, cit., pp. 27-28 y la bibliografía allí indicada).

${ }^{5}$ Nel suo aspetto tal dentro mi fei, qual si fé Glauco nel gustar de l'erba che 'l fé consorto in mar de li altri dèi (vv. 67-70)

Y mi interior su aspecto trasformaba, como Glauco, al gustar marina hierba, consorte de los dioses se tornaba (cit. por la traducción de B. Mitre, Buenos Aires, Latium, 1922, p. 407).

${ }^{6}$ Bostrom, N., op. cit., p. 158. 
efectos incontrolables, así que -según relata Platón- los hombres que gracias al conocimiento construían altares, viviendas y ciudades, "se ultrajaban entre sí por no poseer el arte de la política, de modo que, al dispersarse de nuevo, perecían”. Y por eso Zeus castigó a Prometeo en la roca del Cáucaso, enviando a Hermes, el mensajero de los dioses, para que repartiera el pudor y la justicia entre todos los humanos, a diferencia de las demás artes, ya que en cuanto a valores no puede haber ignorantes conforme a ley de Zeus: "Que todo aquél que sea incapaz de participar del pudor y de la justicia sea eliminado, como una peste, de la ciudad"?.

\section{Viejos dilemas y nuevos retos}

Desde este punto de vista, por tanto, no hay nada nuevo bajo el sol, estaríamos ante un típico debate bioético. La corriente transhumanista milita a favor del uso de las tecnologías emergentes con el fin de mejorar la condición humana ${ }^{8}$. El propio Bostrom la ha descrito recientemente como la posibilidad de mejorar la condición humana física, emocional y cognitiva utilizando el progreso y la tecnología ${ }^{9}$. Sin embargo, la expectación suscitada por los avances en inteligencia artificial plantea un desafío nuevo a la humanidad: no se trata tan solo de la ya metabolizada ambivalencia del progreso científico que caracterizó en su momento el giro bioético, sino de un reto intelectual más profundo. La perspectiva de replicar en máquinas una capacidad de razonamiento similar a la humana, otorgando a estas la facultad de construir representaciones no programadas gracias al aprendizaje proporcionado por algoritmos y Big Data, implicaría pasar el test de Turing, en el que el ordenador triunfa si un evaluador, en preguntas "a ciegas", no logra distinguirlo del humano ${ }^{10}$.

Es cierto, por un lado, que está habiendo mucho debate intelectual previo sobre las aplicaciones derivadas de la inteligencia artificial pero, por el otro, no es menos cierto que no siempre mostramos la suficiente habilidad para prever los escenarios futuribles a partir de la información disponible. Especialmente para vislumbrar los riesgos y las consecuencias potencialmente negativas de las nuevas

\footnotetext{
${ }^{7}$ Platón, Protágoras, ed. bilingüe de G. Bueno, Oviedo, Pentalfa, 1980, 322 a (pp. 125-126).

${ }^{8}$ Hablamos de las llamadas NBIC: Nanotecnología, Biotecnología, tecnologías de la Información y la ciencia Cognitiva.

${ }^{9}$ Entrevista en ABC Cultural, 11 de septiembre de 2018, 85-90.

${ }^{10}$ Ray Kurzweil sitúa en 2029 esta simulación funcional de la inteligencia humana (Kurzweil, R., The Singularity is near: when humans trascend biology, New York, Viking, 2005, p. 199). La 'indistinción' entre el ser humano y el ser artificial a resultas del test de Turing plantearía la consideración de la máquina como agente moral (vid. de Asís, R., Una mirada a la robótica desde los derechos humanos, cit., p. 81 ss.).
} 
aplicaciones científicas. Este sí que es el viejo asunto de la ambivalencia del progreso científico. Al fín y al cabo, también Glauco, una vez asumida la hierba de la inmortalidad, se transformó en una especie de monstruo con cola de pez y fue rechazado por la hermosa ninfa Escila, de la que estaba enamorado. O sea, que toda medalla tiene su reverso: no hay nueva esperanza que no plantee nuevos dilemas.

Por tanto, como simple cautela, convendría tomarse en serio los asuntos de lo transhumano y lo posthumano, al menos como ocasión para reflexionar sobre la evolución de la técnica, incluso en sus versiones más atrevidas, como la de la "singularidad", según la cual el desarrollo exponencial de las nuevas tecnologías emergentes conducirá al superhombre. En esta óptica se han llegado a pronosticar escenarios que conducirían a la inmortalidad -al menos a una élite- gracias a la integración de nuestro conocimiento con los ordenadores. Hay hasta científicos, por ejemplo el biogerontólogo Aubrey de Grey, que mantienen que la primera persona que vivirá mil años ya está viva ${ }^{11}$. Así, cabrían dos orientaciones diferentes dentro de este coacervo de teorías: por un lado estaría el transhumanismo entendido como deseo de mejorar la especie humana a través de la tecnociencia, pero sin forzar rupturas de orden ontológico y sin renunciar a la continuidad con el humanismo clásico; por el otro, se situaría el posthumanismo de la singularidad, sostenido por autores como Kurzweil o el proprio de Grey entre otros, y apoyado por empresas como Google, que alude a una discontinuidad evolutiva con la especie humana y a la creación de una especie nueva, hibridada con máquinas dotadas de una inteligencia artificial tan superior a la nuestra que serían capaces de diseñar máquinas incluso mejores: "Kurzweil sueña con un hombre $<<$ interconectado $>>$ con un ordenador, con todas las redes de internet gracias a implantes cerebrales, que se convertiría así en $<<$ posthumano $>>" 12$.

De todos modos, no hay que olvidar que la tecnología ha influido en la evolución de nuestro cuerpo y nuestra mente desde que hemos construido los primeros utensilios. Me refiero a la $\tau \dot{\varepsilon} \chi v \eta$ (techné) como motor de la historia, desde la pica prehistórica hasta la robótica más sofisticada: lo

11 Harari, Y. N., Homo Deus: a Brief History of Tomorrow, London, Vintage, 2017; Diéguez, A., Transhumanismo. La búsqueda tecnológica del mejoramiento humano, cit., p. 26; Sánchez del Campo Redonet, A., Reflexiones de un replicante legal. Los retos jurídicos de la robótica y las tecnologías disruptivas, Cizur Menor, Aranzadi, 2016, p. 85, quien recuerda que millonarios como Sergey Bin y Peter Thiel, cofundadores respectivamente de Google y Paypal, están financiando la Methuselah Foundation, cuyo creador fue precisamente Aubrey de Grey.

12 Vid. Ferry, L., La revolución transhumanista, Madrid, Alianza Editorial, 2017, p. 42; N. Bostrom, op. cit., p. 167; Llano Alonso, F.H., Homo Excelsior. Los límites ético-jurídicos del Transhumanismo, Valencia, Tirant lo Blanch, 2018, añade a estas dos variedades, que llama respectivamente transhumanismo cultural y transhumanismo cibernético, una tercera, denominada transhumanismo cultural, "inspirado en la crítica postmoderna que realizan autores como Deleuze, Derrida y Foucault contra el humanismo y el proyecto de la modernidad" (p. 28). 
ilustra espléndidamente Stanley Kubrick con la inolvidable metáfora del primer hueso usado como herramienta por un homínido, que se transforma en una astronave del futuro en 2001: una odisea del espacio. Aun teniendo clara esta influencia de la tecnología en la vida humana, el llamamiento a tomar en consideración propuestas, doctrinas y teorías como el transhumanismo, por poco realistas que nos parezcan, deriva de la igualmente comprobada conciencia de que a veces subestimamos las implicaciones presentes y futuras de las innovaciones científicas. Basta con recordar un par de ejemplos iluminantes: el 25 de julio de 1994 la revista Time salía con una portada dedicada al "extraño nuevo mundo de Internet" y en su interior sostenía que "Internet no ha sido diseñado para el comercio"13. Pocos meses después, otra prestigiosísima revista, Newsweek, titulaba un artículo escrito por un físico experto "The Internet?Bah!"14. No se trata solo de equivocarse en una previsión que delata nuestra miopía temporal. Hay en juego mucho más: la inteligencia artificial impactará, está impactando, mucho más de lo que cabe imaginar en todos los ámbitos de la vida y de la producción. Parece que no quedará fuera ni el arte: en los días en que estoy escribiendo estas líneas, precisamente el 25 de octubre de 2018, en la sede neoyorquina de Christie's se ha subastado por primera vez una obra creada por una inteligencia artificial. El retrato de Edmond Belamy lo ha generado un algoritmo y su precio ha alcanzado cotas inesperadas. La base de salida oscilaba entre los siete y los diez mil euros, pero la encarnizada lucha para hacerse con el cuadro ha hecho que se adjudicase por más de 432.500 dólares.

Esto podría parecer anecdótico, pero la preocupación de fondo que impulsa el debate sobre tales asuntos parece evocar las inquietudes epistemológicas que acompañaron la aparición de la inteligencia artificial. Es como si instrumentos como los drones, los coches sin conductor, los robots, los sistemas de armamento autónomo, los virus informáticos, los cíborgs, etc., hubiesen intensificado tanto el mestizaje entre lo humano y lo técnico como para perfeccionar 'sistemas híbridos' y definitivamente, por decirlo en los términos de Alan Turing, "no triviales". Un simple botón de muestra del carácter no descabellado de tales reflexiones: en las elecciones municipales celebradas en abril de 2018 en Tama, uno de los veintitrés distritos que componen la ciudad de Tokyo, uno de los candidatos a la alcaldía ha sido Michihito Matsuda, un androide (robot antropomorfo), que bajo el lema "Artificial Intelligence

\footnotetext{
${ }^{13}$ Elmer-Dewitt, Ph., "The Battle for the Soul of the Internet”, Time, 25 de julio de 1994.

${ }^{14}$ Stoll, C., “The Internet? Bah!”, Newsweek, 27 de julio de 1995.
} 
will change Tama City", ha cosechado 4.013 votos resultando el tercer candidato más votado ${ }^{15}$. Por no hablar de ámbitos más íntimos. Ya son una realidad los robots del sexo: de momento no son máquinas tan sofisticadas, pero están dotadas de una mínima inteligencia artificial y poco a poco la tecnología estará en condiciones de construirlos de un modo cada vez más antropomorfo y capacitados para interactuar respondiendo a estímulos vocales, visuales y táctiles. No solo reconocerán a su usuario, sino que también comprenderán su estado emotivo y aprenderán a identificar sus gustos y preferencias. Las repercusiones psicológicas, sociales, económicas, éticas y jurídicas pueden ser de amplio alcance, hasta el punto de llegar a interrogarse sobre si el sexo con un robot puede integrar los extremos de una infidelidad $^{16}$.

\section{Las implicaciones normativas de las tecnologías convergentes}

De todos modos, por muy sofisticados que sean los sistemas y dispositivos creados hasta ahora, sus capacidades son parciales. No manejan el sentido común y las variables contextuales. Pero, si bien resulta fanta-científica (al menos por ahora) la posibilidad de construir una máquina a la que atribuir responsabilidades de tipo consecuencial por sus acciones, sin que concurra en tales cadenas de actos un ser humano, ya que para ello se requeriría una fiabilidad contrastada y cierto grado de empatía que le permitiera formular un juicio sobre las acciones ajenas, hace ya tiempo que ha sonado la alarma ética y jurídica en las altas esferas. En 2002 se publicó en USA el Informe Converging technologies for improving human performance. Nanotechnology, biotechnology, information technology and cognitive science (CT-NBIC) que explora con optimismo las posibilidades y las promesas de las nuevas aplicaciones tecnológicas de cara a la mejora humana, partiendo de la base de que science must offer society new visions of what it is possible to achieve ${ }^{17}$. Al año siguiente otro informe, Beyond Therapy. Biotechnology and the Pursuit of Happiness, redactado por el Comité de Bioética estadounidense, tomándose en serio el proyecto transhumanista, se muestra mucho más receloso y crítico con la filosofía del perfeccionamiento humano que inspira el informe anterior, y apuesta por limitar el uso de

\footnotetext{
${ }^{15}$ Es posible visitar la página web del candidato, detrás del cual están el vicepresidente de Softbank y un antiguo empleado de Google, en la dirección https://www.ai-mayor.com/ [fecha de consulta: 29 de octubre de 2018].

${ }^{16}$ Acerca de estos temas véase el reciente Balistreri, M., Sex robot. L'amore al tempo delle macchine, Roma, Fandango, 2018.

17 Bond, P. et.al., "Theme A Summary", en M. Roco and W. Bainbridge (eds.), Converging Technologies for Improving Human Performance: Nanotechnology, Biotechnology, Information, Technology and Cognitive Science, NSF/DOC-sponsored report, Springer Science, Dordrecht, 2003, p. 30. Texto disponible en https://www.wtec.org/ConvergingTechnologies/Report/NBIC report.pdf [fecha de consulta: 29 de octubre de 2018].
} 
las NBIC al marco terapéutico ${ }^{18}$. En 2004 también la Unión Europea se hace eco de la insoslayable necesidad de afrontar no solo técnicamente sino también social y políticamente el desafío de las tecnologías convergentes. El informe Converging technologies. Shaping the future of European Societies, también menos indulgente hacia la agenda transhumanista que el primer documento americano, invoca la inclusión de las ciencias sociales y las humanidades dentro del abanico de los saberes "convergentes". De acuerdo con una agenda política más sensible a la tradición humanista europea, el documento apuesta por una mayor integración entre lo social y lo nanotech, en detrimento de la mera optimización técnica del programa transhumanista auspiciado por el primer informe americano $^{19}$. En 2009, en el estudio Human Enhancement, comisionado por el Parlamento Europeo a la Science and Technology Options Assessment (STOA), se reincide en las razones por las cuales la noción de "perfeccionamiento humano", más allá de sus implicaciones técnicas, debe ser compaginada con un más amplio discurso académico y ético-político ${ }^{20}$. Y más recientemente, en 2018, la Comisión Europea ha consignado un marco de reflexión deontológica acerca del actual y futurible impacto de la

18 The President's Council of Bioethics, Beyond Therapy. Biotechnology and the Pursuit of Happiness, Washington D.C., 2003, disponible en https://biotech.law.lsu.edu/research/pbc/reports/beyondtherapy/beyond therapy final_report_pcbe.pdf [fecha de consulta: 29 de octubre de 2018].

${ }^{19}$ Nordmann, A. (ed.), Converging technologies. Shaping the future of European Societies, Conference Report, Unión Europea, 2004, p. 19: "A European approach to CTs needs to be informed by an awareness of their potential and limits. It acknowledges nano-, bio- and info- as key enabling technologies but recognises that only careful agenda-setting can bring them together in viable and socially beneficial convergent research".

20 STOA - Science and Technology Options Assessment, Human Enhancement. Study, IP/A/STOA/FWC/2005-28/SC35, 41 \& 45, European Parliament, $\quad$ May 2009, pp. 16-17. http://www.europarl.europa.eu/RegData/etudes/etudes/join/2009/417483/IPOL-JOIN ET(2009)417483 EN.pdf [fecha de consulta: 29 de octubre de 2018]: "a suitable notion of human enhancement - should not comprise all forms of therapy (if only because of the implications of such a conceptualisation in a health system and policy context) and should take into account that the boundaries between medical treatments and human enhancement are often blurred (for example, when a medical treatment results in an enhancement of the patient's performance, if compared to the patient's performance before occurrence of the treated injury or disease), $\bullet$ should not be based on conceptualisations of normalcy, (dis)ability or health according to which people who were born with certain bodily or cognitive characteristics are deemed as having curable or incurable defects and in which the social and psychological aspects of (dis)abilities and health are ignored, - should be meaningfully limited and therefore exclude such practices as the ordinary use of body-external technological devices, education, physical exercise, mnemonic training, and the consumption of "natural" drugs, such as coca leaves, and food (although these practices can contribute to an enhancement of performance), $\bullet$ should be restricted to the enhancement of individual performance by technoscientific means, and its definition itself should not include the goals of an improvement of the species or a betterment of humanity (although these goals are of utmost importance for the interpretation of the topic of human enhancement, the pertinent debates, and the relevant ideologies). The reasons for us to believe that a notion of human enhancement has to meet these criteria to be suitable for the ethicopolitical and the broader academic discourse on this topic are given in the following sections. We define "human enhancement" as a modification aimed at improving individual human performance and brought about by science-based or technology-based interventions in the human body. This definition includes "strong", second-stage forms of human enhancement with long-term effective or permanent results as well as "temporary" enhancements. Because it is not related to a specific definition of health, this is a non-medical concept of human enhancement. Moreover, we distinguish between purely restorative non-enhancing therapies, therapeutic enhancements and non-therapeutic enhancements". Sobre los cuatro informes, cfr. Hottois, G., "Humanismo, Transhumanismo, Posthumanismo", Revista Colombiana de Bioética, Vol. 8, № 2, 2013, pp. 167-176 y Ferry, L., La revolución transhumanista, cit., pp.22-25. 
Inteligencia Artificial y la Robótica ${ }^{21}$. Las delicadas cuestiones morales originadas por la irrupción de los sistemas high-tech plantean, a juicio del European Group on Ethics in Science and New Technologies, interrogantes del siguiente tenor: ¿cómo proporcionar seguridad y minimizar riesgos en un mundo en el que actúan dispositivos no humanos "autónomos"?; ¿tiene sentido hablar de control y responsabilidad compartida entre el ser humano y las máquinas, es decir, quién responde por los resultados inadecuados provocados por la conducta de estas máquinas inteligentes?; ¿cómo se deberían rediseñar las leyes y los mecanismos institucionales para implementar la gobernanza de un sistema de gestión tecnológica que mantenga como objetivo el bienestar de las personas?; ¿cómo encauzar los procesos democráticos de toma de decisión para hacer frente al problema de un uso de estas técnicas marcado por criterios comerciales o políticos? y last but not least, ¿cómo asegurar la transparencia de estos sistemas en relación con la finalidad pública que, se supone, deberían perseguir? Dicho de otro modo, ¿cómo prevenir la lesión y puesta en peligro de valores básicos como la libertad y la igualdad frente a procesos tecnológicos que manejan, ordenan y clasifican las personas y sus comportamientos con un nivel de sofisticación nunca visto ${ }^{22}$

Tales interrogantes nos conducen a asumir dos premisas, una normativa y la otra empírica. La primera es bien conocida: la formación para el uso de las tecnologías requiere una educación ética y jurídica, además de científica, sin la cual la humanidad no sabría gestionar los resultados del conocimiento. La segunda, a estas alturas de la película, también es pacífica: en un futuro próximo habrá robots muy sofisticados y dotados de inteligencia artificial muy avanzada que podrán tomar decisiones operativas, y ello plantea la cuestión de cómo regular estas capacidades. Huelga recordar, como ejemplo paradigmático en boga hoy en día, el ya archiconocido dilema moral del coche autónomo ante la opción de salvar la vida de los pasajeros o de los peatones ${ }^{23}$. Porque a los ojos del principio tradicional de imputabilidad de las acciones, que reserva la imprevisibilidad a la conducta humana, la impredecibilidad de algunos de estos potentes sistemas cognitivos artificiales adolece de

${ }^{21}$ Por su parte, el Parlamento Europeo aprobó, con fecha de 16 de febrero de 2017, una Resolución con recomendaciones destinadas a la Comisión sobre normas de Derecho Civil sobre robótica, en la que "resulta de vital importancia que el legislador pondere las consecuencias jurídicas y éticas, sin obstaculizar con ello la innovación”. La Resolución incluye una Carta sobre robótica, en la que se propone un código de conducta ética para los ingenieros en robótica, un código deontológico para los comités de ética de la investigación, una licencia para los diseñadores y una licencia para los usuarios (<http://www.europarl.europa.eu/doceo/document/TA-8-2017-

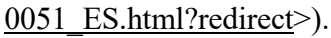

${ }^{22}$ European Group on Ethics in Science and New Technologies, Artificial Intelligence, Robotics and 'Autonomous' Systems, Publications Office of the European Union, Luxemburg, 2018.

${ }^{23}$ Cfr. Cingolani R., Metta, G., Umani e umanoidi. Vivere con i robot, Bolonia, Il Mulino, 2015, pp. 45-48. 
una preocupante opacidad, porque sus acciones ya no están programadas de manera lineal por seres humanos. Tras los algoritmos iniciales establecidos por quienes configuran el funcionamiento del sistema, es imposible saber cómo la máquina conseguirá alcanzar su objetivo mediante operaciones autónomas. Se torna inasequible comprender exactamente cómo pueden los programas de inteligencia artificial AlphaGo aprender a jugar al ajedrez o a otros juegos en pocas horas y superar el nivel de los campeones mundiales humanos o de otros sistemas informáticos ${ }^{24}$. En este sentido sus acciones dejarían de ser inteligibles para el escrutinio humano y pasarían a depender de 'algoritmos inteligentes', una especie de impenetrable caja negra (black box). Dicho de otro modo, se convierten en máquinas que aprenden de la interacción con las personas y por ello adquieren la capacidad de actuar de un modo no previsto inicialmente. No deberíamos olvidar, sin embargo, que estos sistemas basados en redes neuronales artificiales no adquieren por ello una especie de "conciencia discursiva": no pueden dar cuenta del porqué hacen lo que deciden hacer y tampoco un programador podría reconstruir este proceso como si se tratara de un razonamiento ${ }^{25}$.

Este es, en términos generales, el marco de problemas que el transhumanismo plantea en la actualidad. La identificación del rol y de los riesgos entrañados por las tecnologías convergentes aconseja un tratamiento profundo de los aspectos normativos de la interacción entre el ser humano y las aplicaciones basadas en la inteligencia artificial. Las cuestiones afectan tanto a la esfera privada como a la pública, lanzando preguntas de calado jurídico, desde las más clásicas, como ¿quién tiene la responsabilidad por los daños causados por los robots?, ¿cómo impactará en el ámbito laboral el uso extendido de robots inteligentes?, o ¿cómo garantizar el acceso y la disponibilidad de estas tecnologías a todos, sin discriminaciones ${ }^{26}$ ?, hasta las más novedosas, como la relativa a la protección de la privacidad de los individuos: ¿cómo tutelarla cuando, por ejemplo, nos encontremos con un robot dedicado al control de los parámetros médico-clínicos de un anciano para transmitirlos al hospital o a

\footnotetext{
${ }^{24}$ European Group on Ethics in Science and New Technologies, op. cit. Un primer punto de inflexión en las posibilidades de la inteligencia artificial fue la derrota del campeón mundial de ajedrez, Gary Kasparov, frente a Deep Blue, la computadora creada por IBM, en 1997 (cfr. Sánchez del Campo Redonet, A., Reflexiones de un replicante legal. Los retos jurídicos de la robótica y las tecnologías disruptivas, cit., pp. 21-22). El mismo Kasparov reflexiona sobre la asombrosa experiencia en Kasparov, G., Deep Thinking: Where Machine Intelligence Ends and Human Creativity Begins, Nueva York, Public Affairs, 2017.

25 Diéguez, A., “Los profetas ambiguos”, Claves de Razón Práctica, n. 257, marzo/abril 2018, p. 24.

${ }^{26}$ Acerca de las repercusiones de los avances en la ingeniería genética sobre el principio de justicia distributiva, cfr. Buchanan, A., Brock, D. W., Daniels, N., Wikler, D., From chance to choice: genetics and justice, Cambridge University Press, 2002. Como ha señalado R. De Asís, las cuestiones relativas al uso y los límites de la robótica no son nuevas, pues tienen que ver con los problemas bioéticos de autonomía y responsabilidad tradicionalmente suscitados por la reflexión sobre los límites de la investigación científica (de Asís, R., Una mirada a la robótica desde los derechos humanos, cit., p. 75).
} 
su médico en tiempo real ${ }^{27} \mathrm{O}$ dudas jurídicas todavía más radicales: ¿puede un sistema de inteligencia artificial ser considerado persona desde el punto de vista legal? Si la Corte Suprema de EE. UU. ha reconocido a las corporations derechos típicamente reservados a las personas naturales, como la libertad de expresión y la libertad religiosa, al amparo de la Primera y la Decimocuarta enmienda ${ }^{28}$, ¿por qué entonces no podría tener reconocida esa personalidad un programa informático o un robot? Hay especialistas que han mostrado como en USA se puede otorgar actualmente personalidad jurídica a un sistema autónomo, por tanto no humano, sin necesidad de cambiar las leyes, otorgándole el control de una Sociedad Limitada, gracias a la flexibilidad de su estructura legal, que permite su pervivencia temporal aun sin miembros físicos ${ }^{29}$. Naturalmente, al hablar de personalidad jurídica me refiero a la capacidad de ser sujeto portador de derechos, no a la igualación entre el sujeto natural y el sujeto artificial. Obviamente dos empresas no pueden casarse, pero del mismo modo que dos individuos no pueden fusionarse. Sin embargo, descartar por peregrina la eventualidad de reconocer la titularidad de derechos a un ente que carece de rasgos naturales supondría ignorar el significado de "persona" desde el punto de vista jurídico. No se trata de que el derecho, utilizando fórmulas mágicas al estilo de los alquimistas, pueda transformar algo inerte en alguien vivo, sino más bien lo contrario: en el ámbito jurídico la noción de personalidad es una construcción artificial de la ciencia. Me patrocina Kelsen en este punto cuando afirma "que la llamada persona física es una construcción artificial de la ciencia del derecho; que la persona física solo es una persona $<<$ jurídica $>>$ ". Lo que sostiene el posiblemente más ilustre jurista del siglo XX es que el sujeto de derecho no es una entidad física a partir de la cual todo acontece, sino un centro de imputación de derechos y deberes. Más que un 'ser humano' es una unidad

\footnotetext{
${ }^{27}$ Cingolani, R., Metta, G., op. cit., p. 51.

${ }^{28}$ Corte Suprema de los Estados Unidos de América, Citizens United v. Federal Election Commission, Sentencia de 21 de enero de 2010 , disponible en http://www.supremecourt.gov/opinions/09pdf/08-205.pdf; Burwell v. Hobby Lobby Stores, Inc., Sentencia de 30 de junio de 2014, disponible en https://supreme.justia.com/cases/federal/us/573/13-354/case.pdf

${ }^{29}$ Bayern, S., "The Implications of Modern Business-entity Law for the Regulation of Autonomous Systems", Stanford Technology Law Review, Vol. 19: 93, 2015, pp. 93-112: "Consider, then, the following use of an LLC: (1) an individual member creates a membermanaged LLC filing the appropriate paperwork with the state; (2) the individual (along, possibly, with the LLC, which is controlled by the sole member) enters into an operating agreement governing the conduct of the LLC; (3) the operating agreement specifies that the LLC will take actions as determined by an autonomous system, specifying terms or conditions as appropriate to achieve the autonomous system's legal goals; (4) the sole member withdraws from the LLC, leaving the LLC without any members The result is potentially a perpetual LLC - a new legal person - that requires no ongoing intervention from any preexisting legal person in order to maintain its status. To understand the resulting organization's perpetual nature, it is important to recognize that under many modern LLC acts, the sole member's dissociation from the LLC does not require that the LLC terminate its existence. Indeed, many acts specifically contemplate at least the temporary continuation of a memberless LLC because this is a convenient option in several practical cases, such as estate planning. For example, parents might set up an LLC to hold a family's assets and adopt an agreement that states that on the death of the last surviving parent, the couple's children have the option to join the LLC. The modern Uniform Limited Liability Company Actknown as RULLCA - accommodates this possibility, specifically providing that an LLC may by default continue to exist for ninety days without any members" (pp. 100-101).
} 
personificada que consiste en normas que le obligan y facultan: "no se trata de una realidad natural, sino de una construcción jurídica creada por la ciencia del derecho"30.

Este nudo toca además un punto neurálgico del debate general sobre el transhumanismo. Así como las influencias de la moral estoica y del cristianismo añaden a la idea de persona un sentido moral y una fundamentación metafísica, el humanismo tradicional cristaliza los atributos de la persona en el contexto de una naturaleza intangible. La genealogía de los derechos humanos procede de ese marco y el paradigma curativo, no "meliorativo", de la medicina también. De aquí derivan los recelos ante los riesgos planteados por el transhumanismo por parte de destacados autores ubicados, en este debate, entre los "bioconservadores", como Francis Fukuyama. Merece la pena citar directamente sus propias palabras: “(...) Huxley tenía razón (...) la amenaza más significativa planteada por la biotecnología contemporánea estriba en la posibilidad de que altere la naturaleza humana y, por consiguiente, nos conduzca a un estadio «posthumano» de la historia. Esto es importante (...) porque la naturaleza humana existe, es un concepto válido y ha aportado una continuidad estable a nuestra experiencia como especie. Es, junto con la religión, lo que define nuestros valores más básicos. La naturaleza humana determina y limita los posibles modelos de regímenes políticos, de manera que una tecnología lo bastante poderosa para transformar aquello que somos tendrá, posiblemente, consecuencias nocivas para la democracia liberal y para la naturaleza de la propia política" ${ }^{31}$. Es el miedo a que las nuevas tecnologías nos lleven, en cierto modo, a perder nuestra humanidad, nuestra esencia ${ }^{32}$. Desde una perspectiva también esencialista de la naturaleza humana, por citar a otro destacado filósofo y, como Fukuyama, miembro del Comité que redactó el informe estadounidense de 2004 Beyond Therapy, Michael Sandel defiende la ética de la humildad y de la gratitud, que reconoce el carácter de don de los logros y las capacidades humanas, frente a la ética transhumanista del perfeccionamiento, que trasuda hybris y "olvida que la libertad consiste en cierto sentido en una negociación permanente con lo recibido" 33 . Tales posturas, al igual que la de Habermas ${ }^{34}$, ponen al descubierto los nudos gordianos del

\footnotetext{
${ }^{30}$ Kelsen, H., Teoría pura del derecho, $7^{\text {a }}$ ed., Buenos Aires, Porrúa, 1993, pp. 182-184. Y aunque no sea esta quizá la sede adecuada, no faltan argumentos para extender esta perspectiva a la noción de persona más allá del ámbito jurídico. Vid al respecto, desde un punto de vista histórico y antropológico, Mauss, M., Sociología y antropología, Madrid, Tecnos, 1979; Bueno, G., El sentido de la vida. Seis lecturas de filosofía moral, Oviedo, Pentalfa, 1996, en particular pp. 116-122. Para una síntesis de esta cuestión me permito remitir a Campione, R., La teoría social de Anthony Giddens. Una lectura crítica desde la teoría jurídica, Madrid, Dykinson, 2005 , p. 184 ss.

${ }^{31}$ Fukuyama, F., El fin del hombre, Barcelona, Ediciones B, 2002, p. 22

${ }^{32}$ Ibid., p. 165.

${ }^{33}$ Sandel, M., Contra la perfección. La ética en la era de la ingeniería genética. Barcelona: Marbot, 2007, p. 127.
} 
debate sobre las propuestas transhumanistas y las cuestiones prácticas que acarrean: por un lado, el problema de la identificación de los límites entre la aplicación terapéutica o meramente curativa de las nuevas tecnologías convergentes, y su utilización en función de la mejora del individuo (human enhancement). Por el otro, la medida en que estas innovaciones pueden afectar al principio de igualdad y de justicia distributiva, oscilando entre una visión optimista de emancipación humana y una mirada pesimista hacia una nueva sociedad clasista. No parece probable, aun dando respuesta a estos interrogantes, que el asunto se pueda abordar de forma satisfactoria simplemente a través de la labor legislativa, pues el linde entre lo terapéutico y lo meliorativo es fluctuante y difuso. Además, como se ha puesto en evidencia, puede haber mejoras que no tengan argumentos morales de peso en su contra como para considerarlas indeseables; por ejemplo, el aumento de la agudeza visual o el mantenimiento cartilaginoso de las articulaciones en edades avanzadas ${ }^{35}$. A este respecto, Bostrom ha señalado que habría que distinguir las mejoras que ofrecen solo ventajas posicionales, es decir, las que otorgan una ventaja únicamente en la medida en que otros carecen de ella (e.g. un aumento de altura), de las mejoras que aportan beneficios intrínsecamente positivos, como podría ser la mejora del sistema inmunitario o del funcionamiento cognitivo. Solo las del segundo tipo deberían ser promovidas. De ahí que este autor reivindique la urgencia moral de desarrollar medios para revertir el proceso de envejecimiento y proponga así una ampliación del concepto de dignidad humana hasta una "dignidad posthumana"36.

No sorprende, por tanto, que cobre interés el debate relativo a si y cómo pueden quedar afectados conceptos normativos como el de persona o de dignidad humana, pues el reconocimiento de la titularidad de derechos a sistemas tecnológicos ha llegado hasta la polémica suscitada por la concesión de la ciudadanía, por parte de Arabia Saudí el 25 de octubre de 2018, a un robot llamado Sophia, que por ello disfrutaría, en aquel país, de más derechos que los garantizados a las mujeres "de verdad"37. Y este agravio comparativo nos lleva incluso a darle la vuelta al tema, pues estamos

\footnotetext{
${ }^{34}$ Habermas, J., El futuro de la naturaleza humana. ¿Hacia una eugenesia liberal?, Barcelona, Paidós, 2009.

${ }^{35}$ Diéguez, A., "Los profetas ambiguos”, cit., p. 29.

${ }^{36}$ Bostrom, N., "A History of Transhumanist Thought", cit., p. 179; Id, "Human genetic enhancements: A transhumanist perspective", Journal of Value Inquiry, 37 (4), 2003, pp. 493-506; Id., "The fable of the dragon-tyrant", Journal of medical ethics, 31 (5), 2005, pp. 273-277; Id., "The future of human evolution", en Ch. Tandy (ed.), Death and anti-death, Ria University Press, 2005.

37 Hatmaker, T., "Saudi Arabia bestows citizenship on a robot named Sophia", Techcrunch, 26 de octubre de 2017, disponible

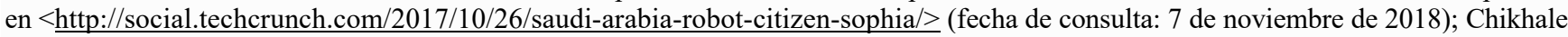
S. N., Vijayrao Gohad, D., "Multidimensional Construct About The Robot Citizenship Law's In Saudi Arabia", International Journal of
} 
planteando la cuestión de si las máquinas podrían adquirir rasgos humanos, pero, por decirlo con las palabras de Alan Turing en la radio en 1951, "si una máquina puede pensar, es posible que piense de manera más inteligente que nosotros, y entonces ¿dónde nos coloca eso?” Parece que, al imaginar un mondo poblado de sistemas artificiales autónomos, es el ser humanos el que se va volviendo menos autónomo. Porque otro factor a tener en cuenta es que con los algoritmos actuales de recopilación de la información entramos en la googlelización de la identidad, de modo que las personas tienden a ser clasificadas por sus gustos y propensiones más que por sus acciones. Gracias al desarrollo del internet de las cosas los datos relativos a nuestra vida cotidiana, a nuestros movimientos tanto físicos como en la red, quedan recogidos de forma digital y son susceptibles de uso comercial mediante la aplicación de ciertos algoritmos $^{38}$. Las empresas tecnológicas que registran nuestra actividad personal en la web utilizan esa información para dirigir la atención del usuario hacia determinadas noticias y anuncios. Así, en las redes sociales, la publicidad es distribuida a través de algoritmos que la envían de forma segmentada a partir de nuestros patrones de navegación. Además, más allá del ámbito comercial, los peligros derivados de un uso sesgado de tal volumen de información son evidentes: en Estados Unidos están de moda programas predictivos de delitos mediante determinados algoritmos que procesan datos históricos individuales para diseñar mapas de delincuencia en las ciudades. En algunos de estos modelos, para calcular los índices, se pregunta por los antecedentes penales de amigos y familiares o por la primera vez que se tuvo trato con la policía; obviamente las respuestas a estas cuestiones serán diferentes según la extracción social del sujeto, pues un recluso de clase media o alta no contestará igual que el procedente de un barrio pobre con más altos niveles de marginación social ${ }^{39}$. Aparte de los graves sesgos sociales o racistas, hay estudios que sostienen que estos programas predictivos, como el

Innovative Research and Advanced Studies (IJIRAS), Vol. 5, N. 1, 2018, pp. 106-108; van Wynsberghe A., Robbins, S., "Critiquing the Reasons for Making Artificial Moral Agents", Science and Engineering Ethics, 2018, pp. 1-27, https://doi.org/10.1007/s11948-0180030-8.

38 De capitalismo "automático", en el que la autonomía de lo humano cede espacio a una identidad post-humana avalada por la tecnociencia habla Rodotà, S., Vivere la democrazia, Bari-Roma, Laterza, 2018, pp. 136-137; sobre el aspecto jurídico de esta tendencia vid. Agote Eguizábal, R., "Inteligencia artificial, ser humano y derecho", Claves de Razón Práctica, n. 257, marzo/abril 2018, pp. 40-45. Para intentar tutelar la privacidad en este contexto de manejo masivo de información personal, ha entrado en vigor este año el Reglamento General de Protección de Datos (UE) 2016/679.

39 Cfr. "Los algoritmos aumentan las desigualdades sociales", entrevista de S. Pérez Soler a Cathy O’Neil, La Vanguardia, 4 de noviembre de 2018. C. O’Neil es autora de Armas de destrucción matemática. Cómo el Big Data aumenta la desigualdad y amenaza la democracia, Madrid, Capitán Swing, 2018. 
COMPAS, ampliamente utilizado en Estados Unidos, ni siquiera aciertan en sus previsiones más que las personas con escasos conocimientos del sector ${ }^{40}$.

\section{Transhumanismo y salud}

En todo caso, al margen del dudoso empleo de la inteligencia artificial y el Big Data, mantiene su sentido la pregunta general evocada por las mencionadas palabras de Turing: ¿qué es lo que nos hace humanos? Porque la hibridación no es el futuro, es el presente. Ha empezado con las prótesis, los marcapasos, los aparatos acústicos, los exoesqueletos; los medicamentos que mejoran la memoria, la concentración y el humor; las drogas que mejoran el rendimiento; la medicina anti-edad; las operaciones de cambio de sexo: todo esto ya está aquí. La inteligencia artificial aplicada a la sanidad ya ha enseñado su enorme potencial. La digitalización de los historiales ha permitido tomar decisiones de manera más rápida y recibir ayuda consultando a expertos a través de imágenes digitales. Además, los robots podrían llegar a ser cirujanos más precisos y fiables incluso sin la inteligencia artificial avanzada, a la vista de las menores incisiones y pérdidas de sangre en intervenciones realizadas en los últimos años. Hay incluso quien cree que estos sistemas podrían ser los mejores expertos en cierto tipo de diagnósticos ${ }^{41}$. Mil seiscientos hospitales utilizan en Estados Unidos Visual DX, un sistema diagnóstico basado en el Big Data, que proporciona resultados excelentes y, si tuviera cierta autonomía decisional, por ejemplo sobre las terapias a adoptar, los pacientes podrían confiarse a las máquinas sin apenas consultar a un médico ${ }^{42}$. Sin tener en cuenta la importancia primordial que el desarrollo de las NBIC tiene en campos como el militar: el Programa ElectRX de la DARPA (la Agencia de Proyectos de Investigación Avanzados de defensa USA) ha asignado 79 millones de dólares para la neuromodulación, pequeños dispositivos electrónicos que interactúan con el sistema nervioso para curar enfermedades como la depresión o la artritis. Pero parece que en realidad el proyecto apunta a la mejora del rendimiento de los soldados mediante el aumento de su capacidad neuronal

\footnotetext{
${ }^{40}$ Dressel, J., Farid, H., "The accuracy, fairness, and limits of predicting recidivism", Science Advances, Vol. 4, n. 1, 2018, DOI: 10.1126/sciadv.aao5580, disponible en http://advances.sciencemag.org/content/4/1/eaao5580/tab-pdf [fecha de consulta: $17 \mathrm{de}$ noviembre de 2018].

${ }^{41}$ Un estudio holandés de 2015 demostró que el diagnóstico por ordenador del cáncer de próstata utilizando imágenes de resonancia magnética era tan bueno como el humano. Otro estudio en Stanford en 2016 ha revelado que la inteligencia artificial, utilizando imágenes de microscopio, diagnosticaba el cáncer de pulmón mejor que los patólogos (Tegmark, M., Vida 3.0. Que significa ser humano en la era de la inteligencia artificial, Madrid, Taurus, 2018, p. 130).

42 Tuniz, C., Tiberi Vipraio, P., La scimmia vestita. Dalle tribú di primati all’intelligenza artificiale, Roma, Carocci, 2018 , p. 230.
} 


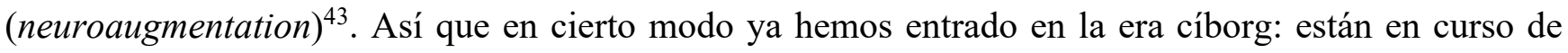
experimentación dispositivos cerebrales para controlar la epilepsia; ojos electrónicos capaces de grabar imágenes; nuevas pieles artificiales, gracias a la nanotecnología, que pueden 'sentir' el frío, el calor y la humedad; auriculares inteligentes que traducen las conversaciones, casi en tiempo real, en cinco idiomas; cuentapasos wifi implantados en el pecho (el primer caso de corazón que forma parte del internet de las cosas); penes biónicos aptos para conseguir una erección instantánea gracias a un interruptor localizado en los testículos; chips electrónicos colocados entre el pulgar y el índice para abrir puertas, acceder a cuentas corrientes, etc.; implantes cocleares que convierten los sonidos en señales eléctricas transmitidas al nervio acústico y después al cerebro ${ }^{44}$.

Naturalmente, no se debe soslayar el reverso de la moneda: las máquinas son creaciones humanas, y también fallan... La máquina canadiense de radioterapia Therac-25 fue diseñada para tratar el cáncer de dos maneras distintas: bien con haces de electrones de baja potencia, bien con uno de alta potencia de rayos $\mathrm{X}$ de varios megavoltios centrado en el objetivo mediante un apuntalamiento especial. Un software sin verificar y con errores provocó que los técnicos aplicasen el segundo procedimiento en lugar del primero, provocando así varias muertes. Entre 2000 y 2001, en el Instituto Oncológico de Panamá, debido a una interfaz de usuario no validada, se suministró durante mucho tiempo cobalto-60 radioactivo. En cualquier caso, según parece, el balance neto aconseja que no cunda el pánico: un reciente informe norteamericano ha establecido que en el período 2000-2013 han ocurrido 144 muertes y 1391 lesiones a causa de la cirugía robótica -por problemas de hardware, chispas eléctricas, piezas rotas o problemas de software por los cuales los aparatos se movían incontroladamente o se apagaban- frente a casi dos millones de operaciones exitosas ${ }^{45}$.

Según la agenda transhumanista, "en un futuro no muy lejano”-citando el íncipit de la película Gattaca - la nanotecnología molecular haría posible eliminar los tumores del tejido sano, así como la contaminación del aire, convertir el carbón en diamantes y la arena en superordenadores.

\footnotetext{
${ }^{43}$ Sánchez del Campo Redonet, A., op. cit., p. 87.

44 Tuniz, C., Tiberi Vipraio, P., op. cit., p. 233.

45 Tegmark, M., op. cit., p. 131. Los casos y los informes mencionados pueden consultarse en Alemzadeh, H. et al., "Adverse Events in Robotic Surgery: A Retrospective Study of 14 Years of FDA Data", PLOS ONE, 11(4), 2016, $<$ https://doi.org/10.1371/journal.pone.0151470>;

$<$ https://web.archive.org/web/20071212183729/http://neptune.netcomp.monash.edu.au/cpe9001/assets/readings/www_uguelph_ca_tgall agh tgallagh.html $>$; $<$ https://www-pub.iaea.org/MTCD/publications/PDF/Pub1114 scr.pdf $>$
} 
Evolucionando, sería de gran ayuda para abolir muchas enfermedades y el envejecimiento ${ }^{46}$. Y de momento ya hay ejemplos actuales de ese futuro mundo biónico poblado de interfaces cerebromáquina, como la Estimulación Electrónica Profunda que se aplica en casos avanzados de depresión o Parkinson. Relata Rafael Yuste, que lidera el proyecto BRAIN impulsado por Barack Obama en 2013 para dibujar el mapa del cerebro, que los pacientes sometidos a esta estimulación mediante un electrodo implantado en el cerebro, cuentan que este tratamiento les cambia la personalidad, en el sentido de que hacen cosas que no hacían antes y esto les lleva a dudar si siguen siendo ellos mismos ${ }^{47}$. Es curioso como esta narración evoca el caso de Phineas Gage, el paciente más famoso de la neurología -gracias a Antonio Damasio- a raíz de un accidente laboral en el cual una barra de hierro le había literalmente atravesado el cráneo sin causarle más consecuencias físicas que el propio agujero. Pese a no haber sufrido daños reseñables, al cabo de un tiempo, se verificaron cambios sensibles en su modo de comportarse: de ser una persona socialmente adaptada pasó a comportare de forma irresponsable e irreverente. Siglo y medio después Damasio, reconstruyendo el caso, mostró que los daños en las zonas prefrontales de la cabeza por las que había pasado el hierro son las responsables del manejo de las emociones y del proceso de toma de decisiones ${ }^{48}$. Si una vara metálica puede modificar aspectos esenciales de la identidad humana, y por tanto la cadena eslabonada de atribuciones que de ella pueden derivar, ¿por qué no podría tener sentido la pregunta acerca de lo que nos diferencia de las máquinas? Considerando que asumen contornos cada vez más realistas las aplicaciones médicas de la inteligencia artificial y las interfaces cerebro-ordenador, mediante la conversión de la actividad neuronal en estímulos que impulsen extremidades robóticas o herramientas externas ${ }^{49}$, en un futuro los nuevos humanos "aumentados" podrían ampliar sus sentidos llegando a ver u oír frecuencias antes inaccesibles o a comunicarse telepáticamente. Amén de escenarios futuristas, quién sabe si poco realistas, las implicaciones para los profesionales de la salud están ya sobre la mesa. Para ellos, dicho de la forma más sencilla y directa posible, ya no se trataría de 'reparar' o de curar sino de mejorar o de 'aumentar' las facultades humanas de individuos sanos conforme a los protocolos de la ciencia médica. Es comprensible que ello suscite una pregunta de carácter ético: ¿es aceptable que sujetos que no padecen

\footnotetext{
${ }^{46}$ Bostrom, N., "A History of Transhumanist Thought”, cit., p. 169.

${ }^{47}$ Yuste, R., Goering, S. et. al., "Four ethical priorities for neurotechnologies and AI", Nature, 9 de noviembre de 2017, p. 162.

${ }^{48}$ Damasio, A., El error de Descartes: la emoción, la razón y el cerebro humano, Barcelona, Crítica, 2001.

49 Piénsese, por ejemplo, en los experimentos de interfaces neuronales cada vez más complejas realizadas en el ámbito del proyecto Cyborg, dirigido por Kevin Warwick, en el cual él mismo se ha implantado un chip conectado a su sistema nervioso para interactuar con un brazo robótico (cfr. Sánchez del Campo Redonet, A., op. cit., p. 87).
} 
enfermedad ni discapacidad alguna puedan mejorar sus prestaciones intelectuales, por ejemplo la memoria, instalándose microchips electrónicos que los conecten a internet por vía inalámbrica? Son escenarios que suenan a la serie Black Mirror, pura ciencia ficción, pero la 'comunidad normativa', tanto la ética como la jurídica, cometería un craso error si se desentendiera sin más de la reflexión sobre estos asuntos so pretexto de su irrealismo. Hasta hace poco habría sonado a fantasía tecnológica la posibilidad de devolver la vista a los ciegos; pero ya es un hecho la implantación de microchips $h i$ tech que pueden devolver cierto grado de visión a los que padecen graves enfermedades genéticas de la retina, como la retinitis pigmentosa. Se trata de un dispositivo de visión artificial muy evolucionado, que permite restaurar la percepción de la luz y de las formas de algunos objetos o personas alrededor, sin que sean necesarios soportes externos como cámaras o gafas ${ }^{50}$. Así pues, también es muy importante el trabajo prospectivo en el ámbito ético y del derecho: los coches totalmente autónomos no son todavía una realidad 'circulante' y, sin embargo, conviene afrontar directamente las cuestiones regulativas que el impacto de este robot podría y podrá producir, en un plazo relativamente breve, en una amplia gama de relaciones sociales, por la sencilla razón de que las normas existentes no contemplan muchas situaciones nuevas. El Reglamento General de Circulación, por ejemplo, obliga a que cada vehículo cuente con un conductor, por tanto en su formulación no podría aplicarse a un coche autónomo $^{51}$. Y estamos hablando de un asunto jurídico que, al fin y al cabo, es de momento marginal. Si tenemos en cuenta que según un informe del British Medical Journal la deficiente atención médica es la tercera causa de muerte en Estados Unidos, con 251.000 fallecimientos al año, por encima de accidentes, enfermedades cardíacas, respiratorias y Alzheimer ${ }^{52}$, podemos hacernos una idea de por qué es más urgente mejorar las aplicaciones de la inteligencia artificial en el ámbito sanitario que en el de los coches autónomos.

En conclusión, si las previsiones tecnocientíficas de la agenda transhumanista se fueran concretando en las próximas décadas, nos toparíamos con una especie de ley de los tres estadios de Comte. El autor de la Filosofía Positiva puso un punto y final a la evolución histórica del 50 Véase, por ejemplo, la intervención recientemente realizada en un hospital italiano, en
https://www.repubblica.it/salute/ricerca/2018/02/05/news/primo impianto di retina artificiale in italia-188086473/

${ }^{51}$ Art. 17.1: "El conductor deberá ser capaz en todo momento de realizar las acciones de conducción necesarias y deberá tener su vehículo bajo control”; vid. Barrio Andrés, M., "El vehículo autónomo y el Derecho", Diario La Ley, Sección Ciberderecho, N²2, 10 de octubre de 2018 .

52 Makary, M.A., Daniel, M., "Medical error - the third leading cause of death in the US", British Medical Journal, 353, 2016, i2139. 
conocimiento: con la sociología, el método científico se completaba y desbancaba por fin a la metafísica, que a su vez se había erigido victoriosa sobre las cenizas de la teología. En este caso, el camino recorrería una línea progresiva que iría del paradigma médico terapéutico al modelo meliorativo del human enhancement, hasta llegar a la condición posthumana de una nueva especie híbrida. Terapia, mejora y superación, estas serían las tres fases de la transición transhumanista. Pero la consumación de este proceso es, una vez más, ciencia ficción, al menos de momento. Mientras tanto, a la luz de lo expuesto, he aquí una invitación a mantener los ojos abiertos sin dejarse llevar por tecnofilias irreflexivas ni prejuicios naturalistas. Adelante, presto, con juicio, decía el astuto Antonio Ferrer en I promessi sposi de Alessandro Manzoni ${ }^{53}$. En términos actuales, que la robótica, la nanotecnología y la inteligencia artificial vayan a convertir las máquinas en las dominadoras del mundo y los humanos en una especie de segunda clase, sería como decir que un asteroide puede estrellarse contra el planeta Tierra y destruirlo. Puede ser, no es realista creerlo ni es probable pero la NASA vigila y está bien que alguien lo haga. Del mismo modo, pensar y reflexionar con seriedad sobre el transhumanismo no es descabellado, por mucho que haya asuntos más apremiantes con los que lidiar ${ }^{54}$. Lo que sí parece repetirse en este caso, como con innovaciones anteriores, es que la difusión de las tecnologías convergentes conduce a una concentración del poder en manos de quienes detentan el control de su dimensión técnica ${ }^{55}$. Y, aplicada a la tecnociencia, una lógica incontroladamente mercantilista produciría una desigualdad social, incluso con tintes meta-evolutivos que, en lugar de una inocente condición transhumana o posthumana, daría lugar a la definitiva deshumanización de la especie.

Al final de esta reflexión, me vienen a la memoria unas palabras de Nietzsche: "por falta de sosiego, nuestra civilización desemboca en una nueva barbarie". En efecto, la reflexión sosegada sobre el transhumanismo podría ser un buen antídoto contra la barbarie tanto de una sociedad entregada al futuro sin conciencia crítica como de un mundo anclado en un pasado reacio a todo cambio. En realidad, si de verdad quisiéramos seguir la lección del humanismo clásico no deberíamos olvidar, como escribía en las primeras líneas, su legado principal: la dimensión histórica de los acontecimientos.

\footnotetext{
${ }^{53}$ Manzoni, A., I promessi sposi, La Spezia, Fratelli Melita Editori, 1994, p. 191.

54 Bergstein, B., "The Great AI Paradox", MIT Technology Review, 15 de diciembre de 2017, disponible en < https://www.technologyreview.com/s/609318/the-great-ai-paradox/>, cit en A. Diéguez, "Los profetas ambiguos", cit., p. 25.

${ }^{55}$ Rodotà, S., op. cit., p. 142.
} 
Los esencialismos no suelen ser buenos consejeros en este sentido, pues lo humano y lo transhumano, me parece, solo pueden ser magnitudes recíprocamente relativas no idealizadas. Qué curioso que las palabras de Nietzsche que acabo de citar provengan de su obra Humano, demasiado humano... ${ }^{56}$.

${ }^{56}$ Nietzsche, F., Humano, demasiado humano, Madrid, Akal, 1996, p. 180. La explicación del título, y la razón por la que lo he traído a colación, la encontramos en Ecce homo: "el título [Humano, demasiado humano] dice «donde vosotros veis cosas ideales, veo yo ¡cosas humanas, ay solo demasiado humanas!»” (Nietzsche, F., Ecce homo, Madrid, Alianza, 2013, p. 100). 


\section{Bibliografía}

Abbagnano, N., Storia della filosofía 2. La filosofia moderna: dal Rinascimento all'Illuminismo, Turín, UTET, 1993.

Agote Eguizábal, R., "Inteligencia artificial, ser humano y derecho”, Claves de Razón Práctica, n. 257, marzo/abril 2018, pp. $40-45$.

Alemzadeh, H. et al., "Adverse Events in Robotic Surgery: A Retrospective Study of 14 Years of FDA Data”, PLOS ONE, 11(4), 2016, <https://doi.org/10.1371/journal.pone.0151470>

Alighieri, D., "La divina comedia", traducción de B. Mitre, Buenos Aires, Latium, 1922.

de Asís, R., Una mirada a la robótica desde los derechos humanos, Madrid, Dykinson, 2015.

Balistreri, M., Sex robot. L'amore al tempo delle macchine, Roma, Fandango, 2018.

Barrio Andrés, M., "El vehículo autónomo y el Derecho", Diario La Ley, Sección Ciberderecho, № 22, 10 de octubre de 2018.

Bayern, S., "The Implications of Modern Business-entity Law for the Regulation of Autonomous Systems", Stanford Technology Law Review, Vol. 19: 93, 2015, pp. 93-112.

B. Bergstein, "The Great AI Paradox", MIT Technology Review, 15 de diciembre de 2017, https://www.technologyreview.com/s/609318/the-great-ai-paradox/

Bond, P. et.al., “Theme A Summary”, en M. Roco and W. Bainbridge (eds.), Converging Technologies for Improving Human Performance: Nanotechnology,Biotechnology,Information Technology, and Cognitive Science,NSF/DOC-sponsored report, Springer Science, Dordrecht, 2003.

Bostrom, N., "A History of Transhumanist Thought", Journal of Evolution and Technology, Vol. 14, 2005, pp. 1-25, publicado sucesivamente en Argumentos de razón técnica, n. 14, 2011, pp. 157-191.

Bostrom, N., "Human genetic enhancements: A transhumanist perspective", Journal of Value Inquiry, 37 (4), 2003 , pp. 493506.

Bostrom, N., "The fable of the dragon-tyrant", Journal of medical ethics, 31 (5), 2005, pp. 273-277.

Bostrom, N., "The future of human evolution", en Charles Tandy (ed.), Death and anti-death: Ria University Press, 2005.

Bostrom, N., Entrevista en ABC Cultural, 11 de septiembre de 2018, 85-90.

Buchanan, A., Brock, D. W., Daniels, N., Wikler, D., From chance to choice: genetics and justice, Cambridge University Press, 2002.

Bueno, G., El sentido de la vida. Seis lecturas de filosofía moral, Oviedo, Pentalfa, 1996.

Campione, R., La teoría social de Anthony Giddens. Una lectura crítica desde la teoría jurídica, Madrid, Dykinson, 2005.

Chikhale, S. N., Vijayrao Gohad, D., "Multidimensional Construct About the Robot Citizenship Law's in Saudi Arabia", International Journal of Innovative Research and Advanced Studies (IJIRAS), Vol. 5, N. 1, 2018, pp. $106-108$.

Cingolani, R., Metta, G., Umani e umanoidi. Vivere con i robot, Bolonia, Il Mulino, 2015.

Damasio, A., El error de Descartes: la emoción, la razón y el cerebro humano, Barcelona, Crítica, 2001.

Diéguez, A., Transhumanismo. La búsqueda tecnológica del mejoramiento humano, Barcelona, Herder, 2017.

Diéguez, A., “Los profetas ambiguos”, Claves de Razón Práctica, n. 257, marzo/abril 2018, pp. 22-31. 
Dressel, J., Farid, H., "The accuracy, fairness, and limits of predicting recidivism", Science Advances, Vol. 4, n. 1, 2018, DOI: 10.1126/sciadv.aao5580, http://advances.sciencemag.org/content/4/1/eaao5580/tab-pdf

Eliot, T.S., La aventura sin fin. Ensayos, ed. de A. Jaume, Madrid, Lumen, 2011.

Elmer-Dewitt, Ph., "The Battle for the Soul of the Internet”, Time, 25 de julio de 1994.

European Group on Ethics in Science and New Technologies, Artificial Intelligence, Robotics and 'Autonomous' Systems, Publications Office of the European Union, Luxemburg, 2018.

Ferry, L., La revolución transhumanista, Madrid, Alianza Editorial, 2017.

Fukuyama, F., El fin del hombre, Barcelona, Ediciones B, 2002.

Habermas, J., El futuro de la naturaleza humana. ¿Hacia una eugenesia liberal?, Barcelona, Paidós, 2009.

Harari, Y. N., Homo Deus: a Brief History of Tomorrow, London, Vintage, 2017.

Hatmaker, T., "Saudi Arabia bestows citizenship on a robot named Sophia", Techcrunch, 26 de octubre de 2017, http://social.techcrunch.com/2017/10/26/saudi-arabia-robot-citizen-sophia/

Hottois, G., "Humanismo, Transhumanismo, Posthumanismo", Revista Colombiana de Bioética, Vol. 8, No 2, 2013, pp. 167-192.

Kasparov, G., Deep Thinking: Where Machine Intelligence Ends and Human Creativity Begins, 2017.

Kelsen, H., Teoría pura del derecho, $7^{\text {a }}$ ed., Buenos Aires, Porrúa, 1993.

Kurzweil, R., The Singularity is near: when humans trascend biology, New York, Viking, 2005.

Llano Alonso, F.H., Homo Excelsior. Los límites ético-jurídicos del Transhumanismo, Valencia, Tirant lo Blanch, 2018.

Makary, M.A., Daniel, M., "Medical error - the third leading cause of death in the US", British Medical Journal, 353, 2016, i2139.

Manzoni, A., I promessi sposi, La Spezia, Fratelli Melita Editori, 1994.

Mauss, M., Sociología y antropología, Madrid, Tecnos, 1979.

Nietzsche, F., Ecce homo, Madrid, Alianza, 2013.

Nietzsche, F., Humano, demasiado humano, Madrid, Akal, 1996.

Nordmann, A. (ed.), Converging technologies. Shaping the future of European Societies, Conference Report, Unión Europea, 2004.

O’Neil, C., Armas de destrucción matemática. Cómo el big data aumenta la desigualdad y amenaza la democracia, Madrid, Capitán Swing, 2018.

Platón, Protágoras, ed. bilingüe de G. Bueno, Oviedo, Pentalfa, 1980.

The President's Council of Bioethics, Beyond Therapy. Biotechnology and the Pursuit of Happiness, Washington D.C., 2003, https://www.wtec.org/ConvergingTechnologies/Report/NBIC report.pdf

Rodotà, S., Vivere la democrazia, Bari-Roma, Laterza, 2018.

Sánchez del Campo Redonet, A., Reflexiones de un replicante legal. Los retos jurídicos de la robótica y las tecnologías disruptivas, Cizur Menor, Aranzadi, 2016.

Sandel, M., Contra la perfección. La ética en la era de la ingeniería genética. Barcelona: Marbot, 2007. 
STOA - Science and Technology Options Assessment, Human Enhancement. Study, IP/A/STOA/FWC/2005-28/SC35, 41 \& 45, European Parliament, May 2009, http://www.europarl.europa.eu/RegData/etudes/etudes/join/2009/417483/IPOLJOIN_ET(2009)417483_EN.pdf

Stoll, C., “The Internet? Bah!”, Newsweek, 27 de julio de 1995.

Tegmark, M., Vida 3.0. Que significa ser humano en la era de la inteligencia artificial, Madrid, Taurus, 2018.

Tuniz, C., Tiberi Vipraio, P., La scimmia vestita. Dalle tribú di primati all'intelligenza artificiale, Roma, Carocci, 2018.

Yuste, R., Goering, S. et. al., "Four ethical priorities for neurotechnologies and AI", Nature, 9 de noviembre de 2017, pp. 159-163.

van Wynsberghe, A., Robbins, S., "Critiquing the Reasons for Making Artificial Moral Agents", Science and Engineering Ethics, 2018, pp. 1-27, https://doi.org/10.1007/s11948-018-0030-8. 